\title{
Evidências de validade da Escala de Segurança Alimentar e Nutricional para adolescentes (ESANa)
}

\author{
Evidence of the validity of the Food and Nutritional Security Scale \\ for adolescents (ESANa)
}

Marjorie Rafaela Lima do Vale (https://orcid.org/0000-0002-1384-5710) ${ }^{1}$

Walberto Silva dos Santos (https://orcid.org/0000-0001-6816-0105) ${ }^{2}$

José Airton de Freitas Pontes Junior (https://orcid.org/0000-0003-2045-2461) ${ }^{3}$

Renata Belizário Diniz (https://orcid.org/0000-0002-3757-6789) ${ }^{3}$

Maria Marlene Marques Ávila (https://orcid.org/0000-0002-8511-2524) ${ }^{3}$
${ }^{1}$ Universidade de Alberta. 116 St \& 85 Ave, Edmonton, AB T6G 2R3. Alberta Canadá. marjorievale@ ualberta.ca

${ }^{2}$ Universidade Federal do Ceará. Fortaleza CE Brasil.

${ }^{3}$ Universidade Estadual do

Ceará. Fortaleza CE Brasil.

\begin{abstract}
This study aimed to develop a valid and reliable scale for assessing food and nutritional insecurity, specifically in adolescents. The initial version of the scale consisted of two subscales: perception of food insecurity and perception of nutritional security. The items were submitted to content analysis $(n=4)$ by a group of food and nutrition security experts, and semantic analysis $(n=20)$ by a group of adolescents conveniently sampled from the target population. After adjustments, the final version of the scale was applied to adolescent students $(n=425)$ aged 12 to 18 years $(m=14.32 \pm 0.96 ; C V=6.7 \%)$. A two-factor model was the most appropriate after performing exploratory factor analysis. The subscales showed modest values of the alpha coefficient (0.69 and 0.60 , respectively). Daily consumption of fruits, vegetables and soft drinks was significantly associated with higher scores in the food and nutrition security perception scale. Therefore, it is recommended to combine food access-based items with other aspects related to attitudes and behaviors towards healthy eating in order to achieve a more accurate picture of adolescent's needs and better guide public policies.
\end{abstract}

Key words Validation studies, Food and Nutritional Security, Adolescent
Resumo O objetivo do trabalho foi desenvolver uma escala válida e fidedigna para a avaliação de insegurança alimentar e nutricional especificamente em adolescentes. A versão inicial da escala foi composta por duas subescalas: percepção de insegurança alimentar e percepção de segurança nutricional. Os itens elaborados foram submetidos à análise de conteúdo por peritos em segurança alimentar e nutricional $(n=4)$ e à análise semântica por adolescentes selecionados por conveniência e oriundos da população alvo $(n=20)$. Após ajustes, a versão final da escala foi aplicada junto a adolescentes escolares $(n=425)$ com idade entre 12 e 18 anos $(m=14,32 \pm 0,96 ; C V$ $=6,7 \%)$. Após realização de análise fatorial exploratória, um modelo de dois fatores foi o que se mostrou mais adequado. As subescalas avaliadas apresentaram valores modestos do coeficiente alfa (0,69 e 0,60, respectivamente). Consumo diário de frutas, verduras e refrigerantes se mostrou significativamente associado a escores mais elevados na escala de percepção de segurança alimentar e nutricional. Sugere-se a viabilidade de combinar itens com base no acesso a alimentos com outros relacionados a atitudes e práticas de alimentação saudável de modo a obter um retrato mais aproximado das necessidades dos adolescentes e melhor orientar políticas públicas.

Palavras-chave Estudos de validação, Segurança Alimentar e Nutricional, Adolescente 


\section{Introdução}

A adolescência é considerada um período de progressivo desenvolvimento biológico, cognitivo, social e emocional ${ }^{1}$. Em países em desenvolvimento como o Brasil, não é raro adolescentes estarem vulneráveis à Insegurança Alimentar e Nutricional $(\text { InSAN })^{2}$. Estar em InSAN significa não ter acesso físico ou financeiro a alimentos de qualidade de modo regular ou não ter capacidades que possibilitem a escolha e consumo de alimentos saudáveis que garantam o exercício de uma vida ativa e saudável ${ }^{3}$. Dessa forma, o planejamento de intervenções de promoção de segurança alimentar e nutricional entre adolescentes torna-se fundamental, uma vez que estar em InSAN tem sido associado a efeitos deletérios de curto e longo prazo, incluindo sintomas de desconforto, angústia e tristeza ${ }^{4}$, menor funcionamento psicossocial ${ }^{5}$, ganho de peso e consequentes comorbidades ${ }^{6}$.

Atualmente, existem diversos métodos que possibilitam a estimativa do nível de InSAN ${ }^{7}$. O instrumento mais utilizado para rastrear a InSAN em nível domiciliar baseia-se em uma escala de percepção da insegurança alimentar e nutricional reportada pelo responsável do domicílio. De modo recente, tendo em vista argumentos de que indivíduos dentro de um mesmo domicílio podem vivenciar diferentes níveis de insegurança alimentar ${ }^{8}$, a adaptação e validação dessa escala para uso em nível individual tem sido proposta.

Há, atualmente, referências nacionais e internacionais de estudos de validação e aplicação desse tipo de escala especificamente com adolescentes 9 . Apesar da obtenção de argumentos favoráveis à validade dessa escala entre a população adolescente, uma das críticas relacionadas a sua utilização inclui o fato de que o uso de questões comumente aplicadas entre adultos resultaria em preocupações relacionadas à validade do instrumento e dos seus achados, uma vez que as experiências de InSAN vivenciadas por adolescentes são únicas em termos de conteúdo e contexto ${ }^{10}$. Para além disso, há também críticas relacionadas ao fato de que o conteúdo dos itens apresentados resgata um componente subjetivo relacionado à vivência da fome, do desemprego, dos riscos de necessidades insatisfeitas e da falta de acesso aos alimentos no dia a dia ${ }^{11}$. Essa concepção é, de certo modo, contrária ao entendimento de que a segurança alimentar e nutricional é resultante não somente da disponibilidade e oferta de alimentos, mas também do consumo desses alimentos, de forma a garantir um estado nutricional ade- quado $^{12}$ e necessário para uma vida ativa e saudável.

Dessa forma, o desenvolvimento de escalas de percepção focado em identificar não somente a acessibilidade financeira a alimentos saudáveis, mas também à identificação de atitudes e práticas alimentares, torna-se fundamental para melhor orientar a utilização de recursos empregados em políticas públicas de forma a torná-las mais eficazes. Diante de tais argumentos, foi objetivo deste estudo desenvolver uma escala válida e fidedigna para a avaliação de insegurança alimentar e nutricional especificamente em adolescentes.

\section{Material e métodos}

Trata-se de um estudo metodológico ${ }^{13}$ de elaboração e proposição de uma escala de avaliação de segurança alimentar e nutricional para adolescentes (ESANa).

\section{Amostragem}

Para o cálculo do tamanho amostral foram utilizados os seguintes parâmetros: total de adolescentes do $9^{\circ}$ ano do ensino fundamental matriculados na zona urbana de Fortaleza em 2012 (32.977 alunos), intervalo de confiança de 95\%, erro máximo tolerável de $5 \%$ e prevalência de insegurança alimentar estimada $(21,3 \%)^{14}$. Como o processo de escolha das unidades amostrais foi por conglomerados, multiplicou-se por dois (efeito do design amostral - DEFF) o tamanho inicialmente calculado. Considerando o tamanho amostral estimado e o número de alunos por escola, nove escolas de ensino fundamental (cinco públicas e quatro privadas) foram selecionadas com probabilidade proporcional ao tamanho.

O processo de amostragem por conglomerados foi utilizado por motivo de viabilidade prática (tempo e custo). O procedimento foi realizado em duas etapas: (a) escolha das escolas; (b) seleção das turmas de forma aleatória dentro das escolas. Todos os alunos, de cada turma selecionada, foram convidados a participar do estudo. Além disso, como se trata de uma validação de instrumento, atendeu-se a recomendação de dez ou mais participantes por número de iten $s^{15}$.

\section{Coleta de dados}

Os dados foram coletados no período de maio a agosto de 2014. A ESANa foi administrada coletivamente nas salas de aula com a presença 
de um pesquisador para a resolução de eventuais dúvidas. Foram também entregues aos alunos um formulário de caracterização socioeconômica e do hábito alimentar com adaptações ${ }^{16}$. Os estudantes que manifestaram concordância tiveram seu peso e altura aferidos.

Para a aferição do peso, os adolescentes foram informados sobre a necessidade de tirar qualquer material dos bolsos do uniforme escolar, como também de descalçar meias e sapatos. A balança utilizada foi da marca G-TECH com capacidade de $150 \mathrm{~kg}$ e precisão de 100 gramas. A altura foi aferida com o adolescente posicionado ereto, com os braços estendidos ao longo do corpo, cabeça erguida e olhando para um ponto fixo à altura dos olhos, descalço e com a cabeça livre de adereços ${ }^{17}$. O instrumento utilizado foi o estadiômetro Personal Caprice da marca Sanny. O valor do IMC foi obtido a partir do cálculo do peso em quilogramas dividido pela altura ao quadrado e classificado segundo critério da Organização Mundial da Saúde ${ }^{18}$.

\section{Desenvolvimento do instrumento}

A construção da ESANa foi orientada por uma perspectiva de percepção de insegurança alimentar e nutricional em nível individual. Para a construção dos itens foi realizada uma revisão de literatura sobre o tema e considerados itens de instrumentos já existentes ${ }^{19-21}$. A versão inicial da escala foi composta por duas subescalas, contendo nove e seis itens (Tabela 1), relacionadas à insegurança alimentar e a segurança nutricional. A diferença entre a dimensão alimentar e nutricional foi baseada no delineamento proposto pela Ação Brasileira pela Nutrição e Direitos Humanos (ABRANDH), no qual a dimensão alimentar está pautada na produção e acesso à alimentos de forma suficiente e estável e a dimensão nutricional incorpora elementos relacionados à escolha $\mathrm{e}$ consumo de alimentos saudáveis ${ }^{22}$.

\section{Análise do conteúdo}

A verificação da adequação do conteúdo foi realizada por um grupo de quatro peritos na área de segurança alimentar e nutricional. A expertise dos participantes foi avaliada utilizando uma modificação dos critérios propostos por Fehring ${ }^{23}$ que incluem a realização de trabalhos, projetos acadêmicos e publicações na área de segurança alimentar e nutricional, totalizando quatorze pontos. Os peritos eram em sua totalidade do sexo feminino, com área de atuação em diferentes localizações geográficas no Brasil (Norte e Nordeste) e no exterior (Canadá), e obtiveram pontuação média de $8 \pm 2,34$ pontos. Os peritos foram selecionados utilizando a técnica bola de neve ${ }^{24}$. Aspectos relacionados a clareza, pertinência prática e relevância teórica foram avaliados utilizando uma escala Likert de 1 a 5 pontos. Os dados obtidos foram utilizados para estimar o Coeficiente de Validade de Conteúdo $(\mathrm{CVC})^{25}$.

\section{Análise semântica}

A compreensão dos itens e das instruções propostas foi avaliada por quatro grupos de adolescentes de escolas públicas e privadas. Objetivou-se manter os grupos homogêneos para evitar polêmicas e constrangimentos ${ }^{26}$. Os grupos foram compostos por cinco a seis adolescentes selecionados por conveniência. Foi solicitado que os adolescentes emitissem opinião sobre a compreensão do formato de resposta e do conteúdo dos itens (por exemplo, "Com suas palavras, o que está sendo perguntado? Há algo que você acha confuso ou difícil de entender?"). Ajustes e correções foram realizados ao final de cada grupo. Novos grupos foram conduzidos até que nenhuma nova sugestão fosse proposta pelos adolescentes. Ao final, foram conduzidos um total de três grupos com estudantes de escolas públicas e um grupo com alunos de escolas particulares.

\section{Análise psicométrica}

A adequação da matriz de dados para realização da análise fatorial foi avaliada utilizando dois indicadores: KMO (Kaiser-Meyer-Olkin) ${ }^{27}$ e o teste de esfericidade de Bartellet (BST) ${ }^{28}$. O número de fatores extraídos foi orientado pelos critérios de Kaiser (Eigenvalues) ${ }^{25}$ e Cattell (Screeplot) ${ }^{29}$.

Após a seleção do número de fatores a serem extraídos, efetuou-se uma análise fatorial exploratória (AFE). Para a seleção dos itens, foi considerada carga fatorial $>0,30^{30}$. De modo adicional, foi verificada a correlação item total corrigida dos itens, com seu respectivo fator, e o coeficiente alfa de Cronbach.

Por fim, os escores produzidos foram classificados em baixo, médio e alto, utilizando pontos de corte baseados nos percentis 25, 50 e 75, e a classificação obtida foi correlacionada com variáveis socioeconômicas, de estado nutricional e de consumo alimentar por meio dos testes Kendalltau e Mann-Whitney. O nível de significância 
Tabela 1. Características sociodemográficas $(n=425)$.

\begin{tabular}{|c|c|c|c|}
\hline Variáveis & $\mathbf{N}$ & $\%$ & Média (Desvio padrão) \\
\hline \multicolumn{4}{|l|}{ Sexo } \\
\hline Feminino & 229 & 53,88 & \\
\hline Masculino & 196 & 46,12 & \\
\hline \multicolumn{4}{|l|}{ Idade } \\
\hline $12-14$ & 283 & 66,59 & \\
\hline $15-\mid 17$ & 136 & 32 & $14,32( \pm 0,96)$ \\
\hline$>18$ & 3 & 0,71 & \\
\hline \multicolumn{4}{|l|}{ Escola } \\
\hline Pública & 239 & 56,24 & \\
\hline Privada & 186 & 43,76 & \\
\hline \multicolumn{4}{|l|}{ Escolaridade chefe de família } \\
\hline Analfabeto & 3 & 0,71 & \\
\hline Até a 4 série & 35 & 8,24 & \\
\hline Fundamental completo & 63 & 14,82 & \\
\hline Médio Completo & 112 & 26,35 & \\
\hline Superior Completo & 91 & 21,41 & \\
\hline \multicolumn{4}{|c|}{ Número de pessoas $<$ de 18 anos no domicílio } \\
\hline Até 3 moradores & 392 & 92,24 & \\
\hline De 4 a 6 moradores & 29 & 6,82 & $1,85( \pm 1,10)$ \\
\hline Acima de 7 moradores & 1 & 0,24 & \\
\hline \multicolumn{4}{|l|}{ Classificação ABEP* } \\
\hline A1 & 3 & 0,71 & \\
\hline A2 & 26 & 8,47 & \\
\hline $\mathrm{B} 1$ & 58 & 13,65 & \\
\hline $\mathrm{B} 2$ & 75 & 17,65 & \\
\hline $\mathrm{C} 1$ & 56 & 13,18 & \\
\hline $\mathrm{C} 2$ & 32 & 7,53 & \\
\hline $\mathrm{D}$ & 4 & 0,94 & \\
\hline $\mathrm{E}$ & 0 & 0 & \\
\hline
\end{tabular}

adotado foi de 0,05 . Todas as análises foram realizadas utilizando diferentes pacotes no software R, 1.0.136 - (C) 2009-2016 RStudio, Inc.

Esta pesquisa foi aprovada pelo Conselho de Ética em Pesquisa da Universidade Estadual do Ceará, segundo os preceitos da Resolução 196/96 do $\mathrm{CNS} / \mathrm{MS}^{31}$.

\section{Resultados}

A amostra final foi constituída por 425 adolescentes, sendo a maioria $(53,9 \%)$ do sexo feminino, com idades entre 12 e 18 anos $(\mathrm{m}=14,32 \pm 0,96$, $\mathrm{CV}=6,7 \%)$. As características sociodemográficas são apresentadas na Tabela 1 .

\section{Análise do conteúdo}

Na Tabela 2 são apresentados os valores do coeficiente de validade de conteúdo. Todos os itens avaliados apresentaram-se adequados nos quesitos de pertinência prática e relevância teórica, significando que os itens estavam adequados para medir o construto avaliado na população em questão. Os itens 6 'Como muito em uma refeição porque pode não haver mais no dia seguinte' e 8 'Pulo refeições' foram considerados pouco claros. Optou-se pela reformulação dos itens após a realização da etapa de análise semântica com os adolescentes. 
Tabela 2. Itens iniciais da ESANa submetidos à análise de conteúdo $(\mathrm{n}=4)$.

\begin{tabular}{lccc}
\hline \multicolumn{1}{c}{ Itens } & Clareza & $\begin{array}{c}\text { Pertinência } \\
\text { prática }\end{array}$ & $\begin{array}{c}\text { Relevância } \\
\text { teórica }\end{array}$ \\
\hline Escala de percepção de insegurança alimentar & & & 1,00 \\
Q1. Como refeições saudáveis. & 0,85 & 1,00 & $1,00,95$ \\
Q2. Tenho que trabalhar para conseguir dinheiro para comer. & 0,90 & 0,80 & 0,95 \\
Q3. Passo o dia inteiro sem comer. & 0,80 & 1,00 & 1,00 \\
Q4. Me preocupo com a falta de comida da minha casa. & 1,00 & 0,90 & 0,90 \\
Q5. Tenho fome, mas não tenho o que comer. & 1,00 & 1,00 & 1,00 \\
Q6. Como muito em uma refeição porque pode não haver mais no & $0,75^{1}$ & 0,86 & 1,00 \\
dia seguinte. & & & 1,00 \\
Q7. No dia a dia tenho poucas opções de escolha alimentar. & 0,80 & 0,95 & 0.85 \\
Q8. Pulo refeições. & $0,70^{1}$ & 0.90 & 0.90 \\
Q9. Como na casa de parentes e amigos porque não tenho comida & 1,00 & 0.90 & \\
em casa. & & & 1,00 \\
Escala de percepção de segurança nutricional & 0,85 & 1,00 & 0,80 \\
Q10. Me considero cuidadoso com a minha saúde. & 1,00 & $0,70^{1}$ & 0,80 \\
Q11. Eu leio textos e informações sobre saúde & 0,90 & 0,80 & 0,95 \\
Q12. Leio o rótulo dos alimentos que como. & 0,90 & 0,90 & \\
Q13. Me importo com a qualidade dos alimentos que como. & & & \\
\hline Clareza: considera a linguagem utilizada nos itens tendo em vista a população respondente. Pertinência prática: considera se o \\
item foi avaliado de forma a mensurar o conceito de interesse na população a qual se destina o instrumento. Relevância teórica: \\
considera o nível de associação entre o item e a teoria. O coeficiente de validade do conteúdo (CVC) foi considerado adequado \\
quando superior a 0.80. 'Item abaixo do CVC estabelecido (0,80). \\
Fonte: Elaborado pelos autores. Fortaleza, 2015.
\end{tabular}

\section{Análise semântica}

$\mathrm{Na}$ etapa de avaliação semântica junto aos adolescentes, termos como 'refeições saudáveis' foram descritos como "frutas/legumes/verduras/ uma refeição balanceada”, demonstrando que os adolescentes possuíam um bom entendimento sobre o termo empregado ${ }^{32}$. Entre as reformulações sugeridas, o item 4 foi reformulado para 'A falta de comida da minha casa me causa preocupação', o item 6 para 'Como muito em uma refeição porque pode não haver mais depois', o item 12 foi dividido em dois itens 'Olho a data de validade dos alimentos antes de comer' e 'Leio a informação nutricional que está no rótulo dos alimentos', bem como o item 13 que se desdobrou em 'Importo-me se os alimentos que eu como são saudáveis' e 'Importo-me se os alimentos que eu como estão livres de contaminantes ou sujeiras'.

\section{Análises psicométricas}

A versão final da escala com quinze itens foi então aplicada e os resultados descritos a seguir. Tendo em vista o não funcionamento de algumas categorias de resposta apresentadas (categorias com menos de 5\% das respostas observadas), e de modo a obter uma solução com maior parcimônia, o formato de respostas original de 7 pontos $(1=$ nunca, $2=$ poucas vezes, $3=$ algumas vezes, $4=$ metade das vezes, $5=$ maior parte das vezes, $6=$ muitas vezes e $7=$ sempre) foi reorganizado em uma solução de 4 pontos, em que as categorias adjacentes $4=$ metade das vezes, 5 $=$ maior parte das vezes, $6=$ muitas vezes e $7=$ sempre, foram colapsadas em uma só categoria: 4 $=$ muitas vezes ou sempre.

\section{Análise Fatorial Exploratória}

Os valores observados no teste de Esfericidade de Bartlett $\left[\chi^{2} 2(14)=799,42(\mathrm{p}<0,001)\right]$ e no Kaiser-Meyer-Olkin - KMO $(0,72)$ confirmaram a adequação da matriz para realização de análises fatoriais ${ }^{25}$. O critério de Kaiser identificou a existência de três fatores com valor próprio (eigenvalues) superior a 1,027. O scree plot $t^{29}$ destacou a existência de dois fatores. Tendo em vista o modesto incremento observado na variância explicada pela solução de três fatores e a maior coerência teórica do modelo mais simples, a solução de dois fatores foi escolhida e utilizada nas análises subsequentes. 
A análise fatorial exploratória com rotação varimax foi então realizada, fixando e extração de dois fatores (Tabela 3). A rotação varimax foi escolhida considerando a baixa correlação entre os fatores $(r=-0,09)$. As cargas fatoriais observadas indicaram que primeiro fator agrupou nove itens relacionados à segurança nutricional. $\mathrm{O}$ item 9 "Pulo refeições" foi removido pelo fato de ter apresentado carga fatorial $<0,30$, e incremento de 0,1 no coeficiente alfa se deletado. A sua remoção resultou em um intervalo de cargas fatoriais de $|31|$ e | 82|. A confiabilidade para esta escala foi estimada em 0,69. O segundo fator agrupou seis itens relacionados à insegurança alimentar. As cargas fatoriais iniciais para este fator variaram de $|0,50|$ a $|0,73|$. O alfa de Cronbach para este fator foi estimado em 0,60 , e para o total da escala em 0,62 .

A Tabela 4 resume os pontos de corte utilizados e a distribuição dos adolescentes entrevistados. Os pontos de corte foram delimitados utilizando os valores dos percentis 25,50 e 75 . Por exemplo, um total de 5 pontos na escala de insegurança alimentar foi classificado como bai- xo risco, uma vez que este valor está abaixo do percentil 25 , indicando que a cada 100 adolescentes desta população, apenas 25 terão obtido uma pontuação inferior à 5 pontos.

Conforme pode ser observado na Tabela 5, enquanto cerca de $30 \%$ dos adolescentes apresentaram baixa pontuação na escala de segurança nutricional, apenas 19\% apresentaram risco elevado de insegurança alimentar. Adicionalmente, adolescentes com baixa pontuação na escala de segurança nutricional relataram, de maneira significativa, consumir menos verduras e frutas e mais refrigerantes diariamente, quando comparados aos que obtiveram maior pontuação. $\mathrm{E}$ apesar de não estatisticamente significante, baixa pontuação na escala de segurança nutricional também foi observado entre adolescentes com sobrepeso oriundos de escolas públicas e cujos chefes de família possuíam menor escolaridade. De maneira oposta, adolescentes em alto risco de insegurança alimentar apresentaram menor prevalência de sobrepeso, ainda que de maneira não significativa.

Tabela 3. Cargas fatoriais, correlação item total corrigida e coeficiente alfa dos itens da ESANA ( $\mathrm{n}=425)$.

\begin{tabular}{|c|c|c|c|c|c|}
\hline Itens & F1 & F1 & h2 & RICT & $\begin{array}{l}\text { Alfa se } \\
\text { deletado }\end{array}$ \\
\hline Q18. Importo-me se os alimentos que eu como são saudáveis. & 0,82 & & 0,679 & 0,664 & 0,60 \\
\hline $\begin{array}{l}\text { Q13. Leio a informação nutricional que está no rótulo dos } \\
\text { alimentos. }\end{array}$ & 0,64 & & 0,412 & 0,616 & 0,63 \\
\hline Q16. Importo-me com a qualidade da água que eu bebo. & 0,56 & & 0,334 & 0,548 & 0,65 \\
\hline Q19. Olho a data de validade dos alimentos antes de comer. & 0,52 & & 0,272 & 0,554 & 0,65 \\
\hline Q1. Como refeições saudáveis. & 0,52 & & 0,269 & 0,422 & 0,65 \\
\hline $\begin{array}{l}\text { Q14. Importo-me se os alimentos que eu como estão livres de } \\
\text { contaminantes ou sujeiras. }\end{array}$ & 0,47 & & 0,223 & 0,546 & 0,66 \\
\hline Q12. Eu leio textos ou vejo programas com informações sobre & 0,42 & & 0,179 & 0,541 & 0,66 \\
\hline Q3. Passo o dia inteiro sem comer. & $-0,34$ & & 0,140 & 0,076 & 0,68 \\
\hline Q9. Pulo refeições. & $-0,26$ & & 0,089 & 0,177 & 0,69 \\
\hline Q5. Tenho fome, mas não tenho o que comer. & & 0,73 & 0,555 & 0,589 & 0,53 \\
\hline $\begin{array}{l}\text { Q7. Como muito em uma refeição porque pode não haver } \\
\text { mais depois. }\end{array}$ & & 0,70 & 0,495 & 0,702 & 0,49 \\
\hline Q8. No dia a dia tenho poucas opções de escolha alimentar. & & 0,68 & 0,469 & 0,692 & 0,52 \\
\hline $\begin{array}{l}\text { Q10. Como na casa de parentes e amigos porque não tenho } \\
\text { comida em casa. }\end{array}$ & & 0,63 & 0,434 & 0,428 & 0,58 \\
\hline Q4. A falta de comida da minha casa me causa preocupação. & & 0,60 & 0,361 & 0,654 & 0,56 \\
\hline Q2. Tenho que trabalhar para comer. & & 0,50 & 0,255 & 0,368 & 0,61 \\
\hline Valor próprio & 2,60 & 2,57 & & & \\
\hline Variância proporcional & 0,17 & 0,17 & & & \\
\hline
\end{tabular}

RICT: Correlação item total corrigida. *Itens com correlação $<0.2$ e incremento de 0,1 no valor do alfa foram deletados. Valores em negrito indicam que as cargas fatoriais foram superiores a 0.30 , e, portanto, o item foi mantido no referido fator. Fonte: Elaborado pelos autores. Fortaleza, 2015. 


\section{Discussão}

A estimativa da insegurança alimentar em indivíduos menores de 18 anos é, de maneira recorrente, feita de modo indireto por meio da percepção de um respondente considerado responsável pelo

Tabela 4. Média, desvio padrão e percentis de classificação $(\mathrm{n}=425)$.

\begin{tabular}{lrr}
\hline & $\mathbf{n}$ & \multicolumn{1}{c}{$\%$} \\
\hline Escala de percepção de segurança & & \\
nutricional (M=22,18; dp=3,88) & & \\
$\quad$ Baixo (<20 pontos) & 129 & 30,3 \\
$\quad$ Médio (20 a 25 pontos) & 215 & 50,6 \\
$\quad$ Alto ( $>25$ pontos) & 81 & 19,1 \\
Escala de percepção de insegurança & & \\
alimentar (M=7,95; dp=2,69) & & \\
$\quad$ Baixo (<6 pontos) & 197 & 46,3 \\
$\quad$ Médio (6 a 9 pontos) & 139 & 32,7 \\
$\quad$ Alto ( $>9$ pontos) & 89 & 21 \\
\hline Fonte: Elaborado pelos autores. Fortaleza, 2015. &
\end{tabular}

domicílio. O uso dessa abordagem, no entanto, impossibilita uma associação fidedigna da situação da insegurança alimentar (avaliada em nível domiciliar) identificada com consequências relativas ao consumo alimentar e estado nutricional (nível individual) $^{33}$.

Dessa forma, estudos objetivando medir a insegurança alimentar de forma auto referida por adolescentes têm sido conduzidos e obtido resultados satisfatórios relacionados à validade de construto e fidedignidade ${ }^{6,33}$ inclusive no Brasil ${ }^{9}$. Os instrumentos validados, no entanto, apresentam uma perspectiva limitada do conceito de segurança alimentar, centrada somente no acesso financeiro aos alimentos, e negligenciando o componente nutricional, reforçando o caráter inédito do presente estudo.

No que concerne às características psicométricas da escala apresentada, os valores elevados do coeficiente de validade de conteúdo atribuídos pelos peritos indicaram que os itens estavam conceitualmente relacionados ao fenômeno da

Fonte: Elaborado pelos autores. Fortaleza, 2015.

Tabela 5. Prevalência de insegurança alimentar e segurança nutricional por variáveis sociodemográficas, de consumo e estado nutricional $(n=425)$.

\begin{tabular}{|c|c|c|c|c|c|c|c|c|}
\hline \multirow{2}{*}{$\begin{array}{l}\text { Variáveis sociodemográficas, de } \\
\text { consumo e estado nutricional }\end{array}$} & \multicolumn{4}{|c|}{ Segurança Nutricional } & \multicolumn{4}{|c|}{ Insegurança Alimentar } \\
\hline & Baixo & Médio & Alto & p-valor ${ }^{a}$ & Baixo & Médio & Alto & p-valor ${ }^{\mathrm{a}}$ \\
\hline \multicolumn{9}{|l|}{ Tipo de escola } \\
\hline Pública & 29,3 & 51,5 & 19,2 & $0,66^{\mathrm{b}}$ & 41,4 & 35,9 & 22,6 & $0,03^{\mathrm{b}}$ \\
\hline Privada & 31,7 & 49,5 & 18,8 & & 52,7 & 28,5 & 18,8 & \\
\hline \multicolumn{9}{|l|}{ Escolaridade } \\
\hline Analfabeto & 35,5 & 49,2 & 15,3 & $0,29^{\mathrm{a}}$ & 42,7 & 33,9 & 23,4 & $0,36^{\mathrm{a}}$ \\
\hline Até 4 série & 34,3 & 54,3 & 11,4 & & 40 & 37,1 & 22,9 & \\
\hline Ens. Fund. Completo & 23,8 & 65,1 & 11,1 & & 52,4 & 22,2 & 25,4 & \\
\hline Ens. Médio completo & 29,4 & 42 & 28,6 & & 43,7 & 40,2 & 16,1 & \\
\hline Ens. Superior completo & 27,5 & 51,7 & 20,8 & & 52,7 & 27,5 & 19,8 & \\
\hline \multicolumn{9}{|l|}{ Estado nutricional } \\
\hline Baixo peso & 25 & 75 & 0 & $0,53^{\mathrm{a}}$ & 75 & 25 & 0 & $0,82^{\mathrm{a}}$ \\
\hline Eutrófico & 28,5 & 51,2 & 19,6 & & 44,1 & 34,9 & 21 & \\
\hline Sobrepeso & 32,6 & 50 & 17,4 & & 46,5 & 30,2 & 23,3 & \\
\hline \multicolumn{9}{|l|}{ Consumo diário de frutas } \\
\hline Sim & 28,9 & 50 & 21,1 & $0,009^{\mathrm{b}}$ & 47,9 & 31,6 & 20,5 & $0,09^{\mathrm{b}}$ \\
\hline Não & 40,5 & 57,1 & 2,4 & & 33,3 & 40,5 & 26,2 & \\
\hline \multicolumn{9}{|l|}{ Consumo diário de verduras } \\
\hline Sim & 19,9 & 52,5 & 27,6 & $<0,001^{\mathrm{b}}$ & 50,9 & 27,5 & 21,6 & $0,16^{\mathrm{b}}$ \\
\hline Não & 43,3 & 48,1 & 8,6 & & 40,6 & 39,1 & 20,3 & \\
\hline \multicolumn{9}{|l|}{ Consumo diário de refrigerante } \\
\hline Sim & 32,1 & 52,6 & 15,3 & $0,001^{\mathrm{b}}$ & 47,6 & 32,7 & 19,7 & $0,22^{\mathrm{b}}$ \\
\hline Não & 23,1 & 41,5 & 35,4 & & 41,5 & 32,9 & 25,6 & \\
\hline
\end{tabular}

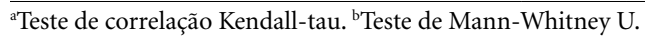

Fonte: Elaborado pelos autores. Fortaleza, 2015. 
insegurança alimentar e nutricional. Empiricamente, a estrutura conceitual proposta foi confirmada tendo em vista que a grande maioria dos itens avaliados apresentou saturações claras em apenas um fator e valores de correlação item total corrigida satisfatórios. Somente os itens "passo o dia inteiro sem comer" e "pulo refeições" foram agrupados em fatores diferentes do originalmente planejado. Isto pode ser justificado pelo fato de comportamentos de restrição alimentar serem relativamente comuns entre adolescentes por motivos além do acesso físico ou financeiro a alimentos, justificando a relevância de medidas de segurança e alimentar e nutricional redesenhadas para esse contexto.

Por exemplo, Panigassi et al. ${ }^{34}$, em um inquérito populacional realizado em 2003, com amostra representativa de famílias de diferentes níveis sociais da cidade de Campinas, constatou que, mesmo nas famílias em segurança alimentar, ou seja, que tinham garantia de acesso aos alimentos, o consumo alimentar era qualitativamente inadequado, onde cerca de $26,3 \%$ dos entrevistados não comiam sequer uma fruta diariamente. Também foi encontrada uma relação inversa entre consumo de doces e refrigerantes e insegurança alimentar, sendo menor o consumo entre famílias de maior insegurança alimentar. Entre a população adolescente, um estudo com 573 escolares do Rio Grande do Sul mostrou associação entre conhecimentos em nutrição e práticas alimentares e obesidade, indicando que crianças com menos conhecimentos e práticas alimentares menos saudáveis tiveram cinco vezes mais chances de serem obesas $(\mathrm{OR}=5,3 ; 1,1-24,9)^{35}$. Dessa forma pode-se perceber que tanto acesso a alimentos saudáveis, quanto conhecimentos e atitudes relacionados a alimentação interferem no consumo e estado nutricional de indivíduos.

Com relação à consistência interna, as subescalas avaliadas apresentaram valores modestos do coeficiente alfa $(0,69 ; 0,60)$ e inferiores ao valor normalmente desejado $(0,70)$. Motivos para a baixa confiabilidade encontrada na escala de segurança alimentar podem estar relacionados à baixa variabilidade nas respostas dos participantes e ao reduzido número de itens utilizados. Adicionalmente, valores de alfa superiores a 0,60 têm sido considerados suficientes em questionários recém-construídos ${ }^{36}$ possivelmente por uma antecipação de problemas relacionados à definição e à compreensão do instrumento pelos participantes. Dessa forma, sugere-se a realização de adicionais entrevistas cognitivas com adolescentes de modo a solucionar potenciais problemas relacionados à compreensão dos itens ou do formato de resposta proposto, e maximizar a qualidade das informações obtidas ${ }^{37}$. Outros estudos que avaliaram a confiabilidade de escalas de insegurança alimentar para adolescentes encontraram valores de alfa de $0,77^{6}$ e $0,48^{33}$.

\section{Conclusão}

Verifica-se que as características psicométricas da ESANa apresentam valores dentro dos padrões exigidos, indicando, portanto, a viabilidade de combinar itens com base no acesso a alimentos com outros relacionados a atitudes e práticas de alimentação. A realização deste estudo foi pautada na necessidade de fornecer um instrumento para melhor orientar a elaboração de políticas e programas de promoção de segurança alimentar e nutricional, uma vez que a proposição de escalas específicas para os componentes alimentar e nutricional permitem a identificação de áreas estratégicas de atuação. Classificação de adolescentes em alto risco de insegurança alimentar indica a necessidade de implementação de programas ou estratégias emergenciais de enfrentamento da fome. Classificação de adolescentes em baixa percepção de segurança nutricional sugere a necessidade de programas educativos que promovam conhecimentos, atitudes e práticas condizentes com adoção de alimentação saudável.

Apesar dos méritos da ESANa, algumas limitações podem ser mencionadas. Primeiro, o fato de terem sido coletados dados de alunos de nove escolas, de cinco das seis regiões administrativas da cidade, de diferentes estratos socioeconômicos, não foi suficiente para garantir uma maior heterogeneidade da amostra, limitando dessa forma qualquer generalização. Segundo, a confiabilidade do instrumento também foi inferior ao desejado, sugerindo-se a realização de adicionais entrevistas cognitivas com adolescentes, que permitam solucionar potenciais problemas relacionados à compreensão dos itens ou do formato de resposta adotado. 


\section{Colaboradores}

MRL Vale: responsável pela concepção e desenho do estudo, coleta, análise e interpretação dos dados, escrita, revisão e aprovação final do manuscrito. WS Santos: responsável pela revisão do desenho do estudo, da análise e da interpretação dos dados, e revisão e aprovação final do manuscrito. JA Pontes Junior: responsável pela revisão da análise e da interpretação dos dados, e revisão e aprovação final do manuscrito. RB Diniz: responsável pela revisão e aprovação final do manuscrito. MMM Ávila: responsável pela revisão do desenho do estudo e da interpretação dos dados, revisão e aprovação final do manuscrito.

\section{Agradecimentos}

Os autores agradecem à Coordenação de Aperfeiçoamento de Pessoal de Nível Superior pela bolsa de mestrado concedida. Agradecem também à Secretaria Municipal de Educação do município de Fortaleza e aos diretores e coordenadores das escolas participantes pela colaboração.

\section{Referências}

1. Stang J, Story M. Adolescent Growth and Development. In: Story M, Stang J, organizadores. Guidelines for adolescent nutrition services. Minneapolis: Center for Leadership, Education and Training in Maternal and Child Nutrition, Division of Epidemiology and Community Health, School of Public Health, University of Minnesota; 2005. p. 1-8.

2. Silva GLD, Espinosa MM, Bezerra ACD, Guimarães LV, Lima-Lopes MA. Insegurança alimentar em domicílios com adolescentes da Amazônia Legal Brasileira: prevalência e fatores associados. Cad Saúde Pública 2013; 29(2):335-348.

3. Organização das Nações Unidas para Alimentação e Agricultura (FAO). Declaração de Roma Sobre a Segurança Alimentar Mundial e Plano de Acção da Cimeira Mundial da Alimentação. Roma: World Food Summit; 1996.

4. Widome R, Neumark-sztainer D, Hannan PJ, Haines J, Story M. Eating when there is not enough to eat: eating behaviors and perceptions of food among food -insecure youths. Am J Public Health 2009; 99(5):822828.

5. Casey PH, Szeto KL, Robbins JM, Stuff JE, Connell C, Gossett J, Simpson PM. Child health-related quality of life and household food security. Arch Pediatr 2005; 159(1):51-56.

6. Gulliford MC, Mahabir D, Nunes C, Rocke B. Self-administration of a food security scale by adolescents: item functioning, socio-economic position and food intakes. Public Health Nutr 2005; 8(7):853-860.

7. Jones AD, Ngure FM, Pelto G, Young SL. What Are We Assessing When We Measure Food Security? A Compendium and Review of Current Metrics. Advances Nutr 2013; 4(5):481-505.

8. Bickel G, Nord M, Price C, Hamilton W, Cook J. Guide to measuring household food security in the United States: Revised 2000. Washington: US Department of Agriculture, Food and Nutrition Service; 2000.

9. Coelho SEAC, Vianna RFT, Segall-Correa AM, Perez-Escamilla R, Gubert, MB. Insegurança alimentar entre adolescentes brasileiros: um estudo de validação da Escala Curta de Insegurança Alimentar. Rev Nutr 2015; 28(4):385-395.

10. Fram MS, Frongillo EA, Draper C, Fishbein E. Development and validation of a child-report assessment of childhood food insecurity and comparison to parent-report assessment. Mississipi: Southern Rural Development Center; 2012.

11. Oliveira JS, Lira PIC, Veras ICL, Maia SR, Lemos MCC, Andrade SLLS, Viana Junior MJ, Pinto FCL, Leal VS, Batista Filho M. Estado nutricional e insegurança alimentar de adolescentes e adultos em duas localidades de baixo índice de desenvolvimento humano. Rev Nutr 2009; 22(4):453-465.

12. Aker J, Lemtouni A. A framework for assessing food security in face of globalization: the case of Morroco. Agroalimentaria 1999; 8(13):13-26.

13. Polit D, Beck CT, Hungler B. Fundamentos de pesquisa em enfermagem: métodos, avaliação e utilização. Porto Alegre: Artmed; 2004.

14. Instituto Brasileiro de Geografia e Estatística (IBGE). Pesquisa Nacional por Amostra de Domicílio - PNAD 2009. Rio de Janeiro: IBGE; 2009. 
15. Hair JR, Black WC, Babin BJ, Anderson RE, Tatham RL. Multivariate Data Analysis. 6a ed. Upper Saddle River: Pearson Prentice Hall; 2006.

16. Segall-Corrêa AM, Pérez-Escamilla R, Maranha LK, Sampaio MFA, Yuyama L, Alencar F, Vianna RPT, Vieira ACF, Coitinho D, Schmitz BS, Leão MM, Gubert M. Acompanhamento e avaliação da segurança alimentar de famílias brasileiras: validação de metodologia e de instrumento de coleta de informação. Campinas: Universidade Estadual de Campinas, Organização Pan-Americana da Saúde, MS; 2003. (Relatório Técnico).

17. Brasil. Ministério da Saúde (MS). Secretaria de Atenção à Saúde. Departamento de Atenção Básica. Orientações para a coleta e análise de dados antropométricos em serviços de saúde: Norma Técnica do Sistema de Vigilância Alimentar e Nutricional - SISVAN. Brasília: MS; 2011.

18. World Health Organization (WHO). Physical status: the use and interpretation of anthropometry: report of a WHO Expert Committee. Geneva: WHO Technical Report Series; 1995.

19. Pérez-Escamilla R, Segall-Corrêa AM, Kurdian ML, Sampaio MFA, Marín-León L, Panigassi G. An Adapted Version of the U.S. Department of Agriculture Food Insecurity Module Is a Valid Tool for Assessing Household Food Insecurity in Campinas, Brazil. $J$ Nutr 2004; 134(8):1923-1928.

20. Smith LC, Obeid AEE, Jensen HH. The geography and causes of food insecurity in developing countries. Agric Econ 2000; 22(2):199-215.

21. Jayanti RK, Burns AC. The antecedents of preventive health care behavior: An empirical study. J Acad Mark Sci 1998; 26(1):6-15.

22. Burity V, Franceschini T, Valente F, Recine E, Leão M, Carvalho MF. Direito humano à alimentação adequada no contexto da segurança alimentar e nutricional. Brasília: ABRANDH; 2010.

23. Fehring RJ. Methods to validate nursing diagnoses. Heart Lung 1987; 16:6.

24. Handcock MS, Gile KJ. On the Concept of Snowball Sampling. Sociol Methodol 2011; 41(1):367-371.

25. Hernández-Nieto RA. Contributions to statistical analysis. Mérida: Universidade Los Andes; 2002.

26. Dias CA. Grupo Focal: Técnica de coleta de dados em pesquisas qualitativas. Inf Soc Est 2000; 10(2):7-22.

27. Kaiser HF. The Application of Electronic Computers to Factor Analysis. Educ Psychol Meas 1960; 20(1):141151.

28. Dziuban CD, Shirkey EC. When is a correlation matrix appropriate for factor analysis? Some decision rules. Psychological Bulletin 1974; 81(6):358-361.

29. Cattell RB. The scree test for the number of factors. Multivar Behav Res 1966; 1(1):245-276.
30. Tabachnick BG, Fidell LS, Osterlind SJ. Using multivariate statistics. New York: Pearsons; 2001.

31. Brasil. Ministério da Saúde (MS). Conselho Nacional de Saúde (CNS). Resolução no 196 de 10 de outubro de 1996. Diretrizes e Normas Regulamentadoras de Pesquisas Envolvendo Seres Humanos. Diário Oficial da União 1996; 16 out.

32. Toral N, Conti MA, Slater B. A alimentação saudável na ótica dos adolescentes: percepções e barreiras à sua implementação e características esperadas em materiais educativos. Cad. Saúde Pública 2009; 25(11):2386-2394.

33. Connell CL, Nord M, Lofton KL, Yadrick K. Food Security of Older Children Can Be Assessed Using a Standardized Survey Instrument. J. Nutr 2004; 34(10):2566-2572.

34. Panigassi G, Segall-corrêa AM, Marin-León L, Pérez-Escamilla R, Maranha LK, Sampaio M FA. Insegurança alimentar intrafamiliar e perfil de consumo de alimentos. Rev Nutr 2008; 21:135-144.

35. Trichesa R, Giuglianib ERJ. Obesidade, práticas alimentares e conhecimentos de nutrição em escolares. Rev Saúde Pública 2005; 39(4):541-547.

36. Nunnally JC. Psychometric theory. 2a ed. New York: McGraw-Hill; 1978.

37. Pergher GK, Stein LM. Entrevista cognitiva e terapia cognitivo-comportamental: do âmbito forense à clínica. Rev Bras Ter Cogn 2005; 1(2):11-20.

Artigo apresentado em 21/08/2018

Aprovado em 15/04/2019

Versão final apresentada em 17/04/2019

Editores chefes: Romeu Gomes, Antônio Augusto Moura da Silva 